$i=1, \cdots, c$, and $c \leqq 2, r_{1}$ or $r_{2}=1$. But this implies that $A$ is a permutation matrix.

Conjecture. If $A=\left(a_{i j}\right)$ is an $n$-square $(0,1)$-matrix then

$$
p(A) \leqq \prod_{i=1}^{n}\left(r_{i} !\right)^{1 / r_{i}}
$$

with equality if and only if there exist permutation matrices $P$ and $Q$ such that $P A Q$ is a direct sum of matrices all of whose entries are 1.

The conjecture is known to be true for all $(0,1)$-matrices whose row sums do not exceed 6 .

\title{
REFERENCE
}

1. H. J. Ryser, Combinatorial mathematics, Carus Math. Monograph No. 14, Math. Assoc. Amer., 1963.

UNIVERSITY OF FLORIDA

\section{THE COLLINEATION GROUPS OF DIVISION RING PLANES. I. JORDAN ALGEBRAS}

BY ROBERT H. OEHMKE AND REUBEN SANDLER

Communicated by Nathan Jacobson, July 17, 1963

In this note, we outline a method which reduces the determination of the collineation group of a division ring plane to the solution of certain algebraic problems-in particular, to the question of when two rings of a certain type are isomorphic. This method is then applied to planes coordinatized by finite dimensional Jordan algebras of characteristic $\neq 2,3$, and their collineation groups are determined. Complete arguments and detailed proofs will appear elsewhere.

1 . Let $\Re$ be a nonalternative division ring, let $\pi(\Re)$ be the projective plane coordinatized by $\Re$, and let $G(\pi)$ be the collineation group of $\pi$. Then (see [1]) $G(\pi)$ possesses a solvable normal subgroup whose structure is known, the elementary subgroup, such that the factor group is isomorphic with the group of autotopisms of $\Re, A(\Re)$. Also, $A(\Re) \approx H(\pi)$, where $H(\pi)$ consists of those elements of $G(\pi)$ which leave fixed the points $(\infty),(0)$, and $(0,0)$. (See [2], Chapter 20 for the coordinatization of projective planes.)

Let $B(\Re)$ be the automorphism group of $\Re$. Then $B(\Re) \approx H_{1}(\pi)$, where $H_{1}(\pi)$ consists of those elements of $H_{1}(\pi)$ which leave the point 
$(1,1)$ fixed. Thus, a coset decomposition

$$
H(\pi)=\sum H_{1}(\pi) \alpha_{i}
$$

can be obtained, and our first result, which is easily proved, is

THEOREM 1. $\phi_{1}, \phi_{2}$ are in the same coset if and only if $(1,1) \phi_{1}$ $=(1,1) \phi_{2}$.

Now, call a pair $(a, b)$ admissible if there is an element of $H(\pi), \alpha$, such that $(1,1) \alpha=(a, b)$. If all admissible pairs can be determined, then we will know what each coset does to the point $(1,1)$, and can actually begin to look for coset representatives.

At this time, we need

THEOREM 2. Let $\Re, \Re^{\prime}$ be the two coordinate rings for a plane defined by the quadrangles $(\infty),(0),(0,0),(1,1)$ and $(\infty)^{\prime},(0)^{\prime},(0,0)^{\prime},(1,1)^{\prime}$, respectively. Then $\Re$ and $\Re^{\prime}$ are isomorphic if and only if there is a collineation $\alpha$ such that $(\infty) \alpha=(\infty)^{\prime},(0) \alpha=(0)^{\prime},(0,0) \alpha=(0,0)^{\prime}$, and $(1,1) \alpha=(1,1)^{\prime}$.

We now recoordinatize $\pi(\Re)$ using the quadrangle $(\infty)^{\prime}=(\infty)$, $(0)^{\prime}=(0),(0,0)^{\prime}=(0,0),(1,1)^{\prime}=(a, b)$. Call the new coordinate ring $\mathfrak{S}_{a, b}$. Then $\Re$ and $\mathfrak{S}_{a, b}$ are isotopic, and Theorem 2 says that $(a, b)$ is an admissible pair if and only if $\Re$ and $\mathfrak{S}_{a, b}$ are isomorphic.

Upon recoordinatizing, we find that $(\Re,+)$ and $\left(\Im_{a, b},+\right)$ are isomorphic under the trivial identification of elements, and that multiplication in $\mathfrak{S}_{a, b}$ can be defined by

$$
x * y=\left\{\left(x R_{a^{-1}}^{-1}\right)\left[\left(\left(y R_{a^{-1}}^{-1}\right)\left(b L_{a}^{-1}\right)\right) L_{a}^{-1}\right]\right\} R_{b L_{a}^{-1}}^{-1} R_{a^{-1}},
$$

where $R_{x}$ and $L_{x}$ represent right and left multiplication in $\Re$.

2. In trying to find all admissible pairs when $\mathfrak{R}$ is a finite dimensional Jordan algebra, one needs to prove the following theorem which is an important tool in the subsequent analysis.

TheOREм 3. The left, middle, and right nuclei of a finite dimensional Jordan division algebra are all equal.

The next step is fairly long and difficult, and consists in using in various subtle ways the assumptions that $\widetilde{S}_{a, b}$ is commutative and satisfies the Jordan identity

$$
R_{x} R_{x^{2}}=R_{x^{2}} R_{x}
$$

until the following result is reached: 
THEOREM 4. If $\Re$ is a finite dimensional Jordan algebra of characteristic $\neq 2,3$, then $(a, b)$ is an admissible pair if and only if $a$ and $b$ are both elements of the center of $\Re$.

Thus, we know not only that $\Im_{a, b}$ and $\Re$ are isomorphic if and only if $a$ and $b$ are in the center of $\Re$, but from (2), we see that

$$
x * y=x y,
$$

which says that the trivial mapping is an isomorphism. But actually knowing an isomorphism between $\Re$ and $\Im_{a, b}$ allows one to write explicitly a set of coset representatives, $\alpha_{a, b}$, for (1). These coset representatives are defined by:

$$
\begin{aligned}
(x, y) \alpha_{a, b} & =(x a, y b) \\
(m) \alpha_{a, b} & =\left(m a^{-1} b\right) .
\end{aligned}
$$

All that remains, then, is to determine the automorphism groups of these Jordan algebras. But this has been done for most of the classes of such algebras, and for a complete account of what is known about the automorphism groups of Jordan algebras, see [3, pp. 190191].

\section{REFERENCES}

1. A. A. Albert, Finite division algebras and finite planes, Proc. Sympos. Appl. Math. Vol. 10, pp. 53-70, Amer. Math. Soc., Providence, R. I., 1960.

2. Marshall Hall, Jr., The theory of groups, Macmillan, New York, 1959.

3. N. Jacobson, Some groups of transformations defined by Jordan algebras. I, J. Reine Angew. Math. 201 (1959), 178-195.

Institute for Defense Analyses 